\title{
DECREASE RADAR CROSS-SECTION OBJECTIVES USING THE METAMERIAL
}

\author{
Aleksandr Mironchev ${ }^{1}$, Vladimir Yakubov \\ National Research Tomsk State University, 634050, Tomsk, Russia
}

\begin{abstract}
In this work influence of an electromagnetic field on lattices from close located ring conductors is shown. RCS is also calculated and shown that at $0 \leq n^{2}<1$ there is the considerable decrease of an object.
\end{abstract}

\section{Introduction}

Efficient scattering area in a radar-location - the area of some dummy even surface located normally to the direction of an incident plane wave and which is the ideal and isotropic reradiate which, being placed in a purpose arrangement point, creates in the location of the antenna of radar station the same fluency of power, as the actual purpose [1].

\section{Experimental study}

RCS is the quantitative measure of property of an object to disseminate an electromagnetic wave. Along with energy potential of a send-receive path and intensification coefficient of antennas of radar station, RCS of an object enters the equation of range of a radar-location and determines the range at which an object can be found by the radar. The increased RCS value means larger radar visibility of an object, decrease in RCS complicates detection. Decreases of RCS of the purposes it is possible with use of composition metamaterial of a radio-frequency range.

On the basis of numerical model operations on interaction of an electromagnetic radiation with structure from elemental annuluses [2, 3], the experimental model of metamaterial is created. The exemplar was executed in the form of matrix structure from the ring conductors located in frothed polystyrene. Distances between parallel ring conductors were made by $0.5 \mathrm{~cm}$ (Figure 1 ).

\footnotetext{
${ }^{1}$ Corresponding author: mironchev42@mail.ru
} 


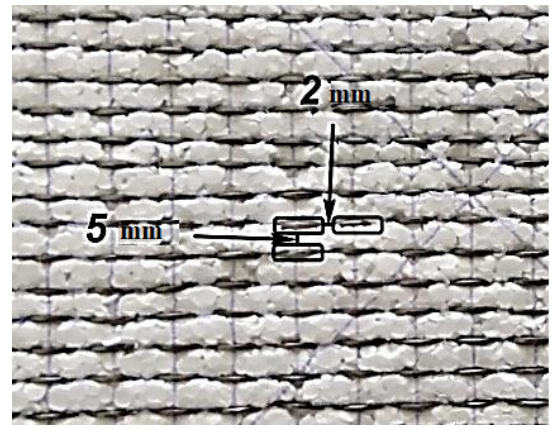

Fig. 1. The metamaterial model from ring conductors (top view).

A series of the pilot studies was made:

1. The composite from ring conductors one layer, thickness is $1 \mathrm{~cm}$.

2. The composite from ring conductors two layers, thickness is $2 \mathrm{~cm}$.

3. A composite from ring conductors three layers, thickness of $3 \mathrm{~cm}$.

4. The composite from ring conductors four layers, thickness is $4 \mathrm{~cm}$.

5. Plexiglass, the thickness of $1.8 \mathrm{~cm}$.

Carrying out an experiment was carried out by method is based on measurement of electrophysical properties in the free space [4].

In the Figure 2 dependences of a phase (1) and amplitude (2) on the frequency of the signal from $0.05 \mathrm{GHz}$ up to $22 \mathrm{GHz}$ which passed through a single-layer lattice from elemental annuluses are presented. Lines (3) and (4) represented the linear regression for a phase (3) and amplitude (4) respectively. Point noted mean values and fiducially intervals. In a superwide frequency band the curve of a phase has linearly the decreasing character that is characteristic of metamaterials.

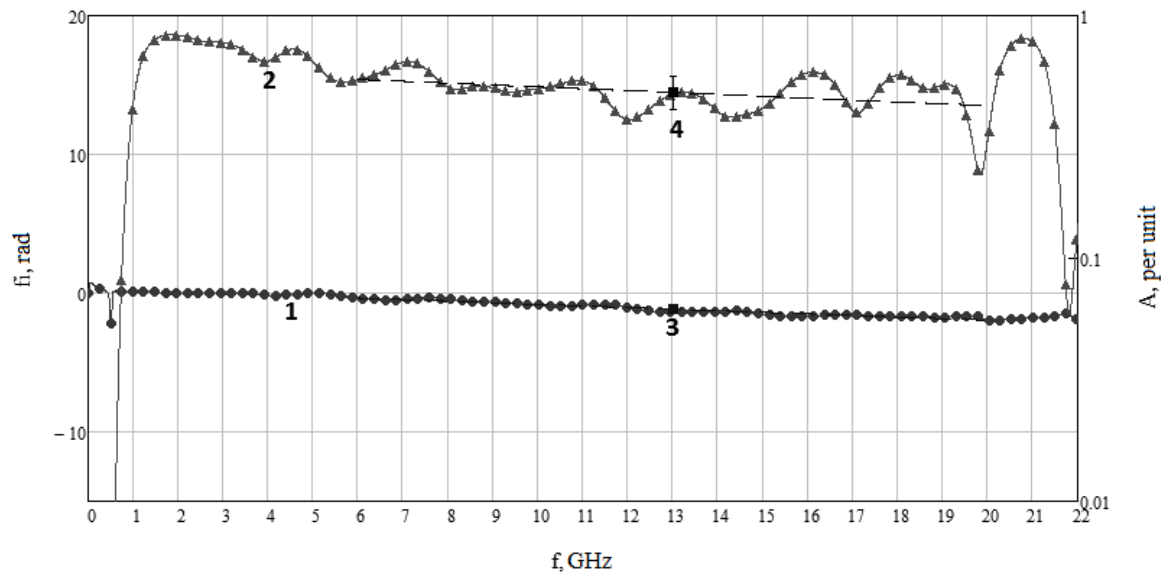

Fig. 2. Dependence of amplitude (2) and phase (1) of transmitted signal on frequency for one layer of rings $1 \mathrm{~cm}$ thick, linear regression of phase (3), linear regression of amplitude (4).

Complex refractive index in the frequency range from $6 \mathrm{GHz}$ to $20 \mathrm{GHz}$ $n=0.46 \pm 0.02+\mathrm{i} 0.09 \pm 0.01$. The result obtained indicates that a single-layered lattice of closed ring elements is capable of reducing the complex refractive index due to the backwash of the wave. As discussed in the previous chapters, this becomes possible due to the appearance of a near field near a spatial lattice in which there is no phase increase, as occurs in the far radiation zone.

Consider the results of an experiment carried out with two layers of Figure 3. You can see, as in the previous case with one layer, the phase of the transmission coefficient is 
linear. Throughout the entire frequency interval, a negative slope of the phase curve is observed, which is characteristic of a metamaterial.

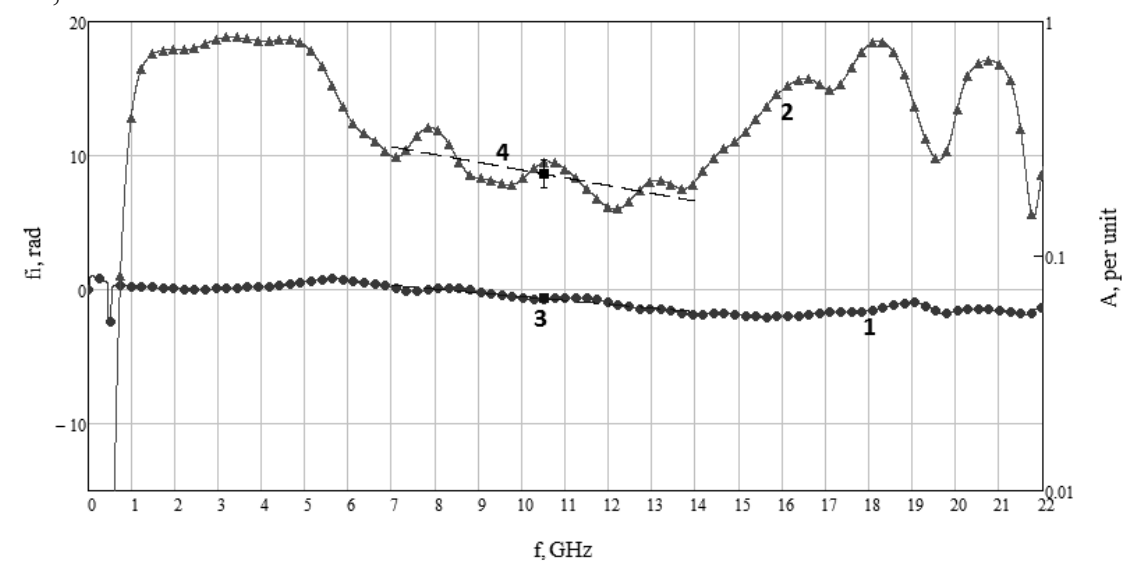

Fig. 3. Dependence of amplitude (2) and phase (1) of transmitted signal on frequency for one layer of rings $2 \mathrm{~cm}$ thick, linear regression of phase (3), linear regression of amplitude (4).

An experiment carried out with two layers showed the result $n=0.35 \pm 0.05+\mathrm{i} 0.14 \pm 0.03$ in the $7 \div 14 \mathrm{GHz}$ band. Such values of the real part of the refractive index indicate an increase in the properties of the metamaterial in a lattice made up of annular conductors. At the same time, the imaginary part of the refractive index begins to increase with the increase in the number of layers.

Next, Figure 4 shows the dependence of the amplitude (2) and phase (1) of the transmitted signal on the frequency for three layers of rings $3 \mathrm{~cm}$ thick. The behavior of the curves is similar to the curves given for two layers with a total thickness of $2 \mathrm{~cm}$. Also, there is a frequency interval on which the phase foray has a negative course.

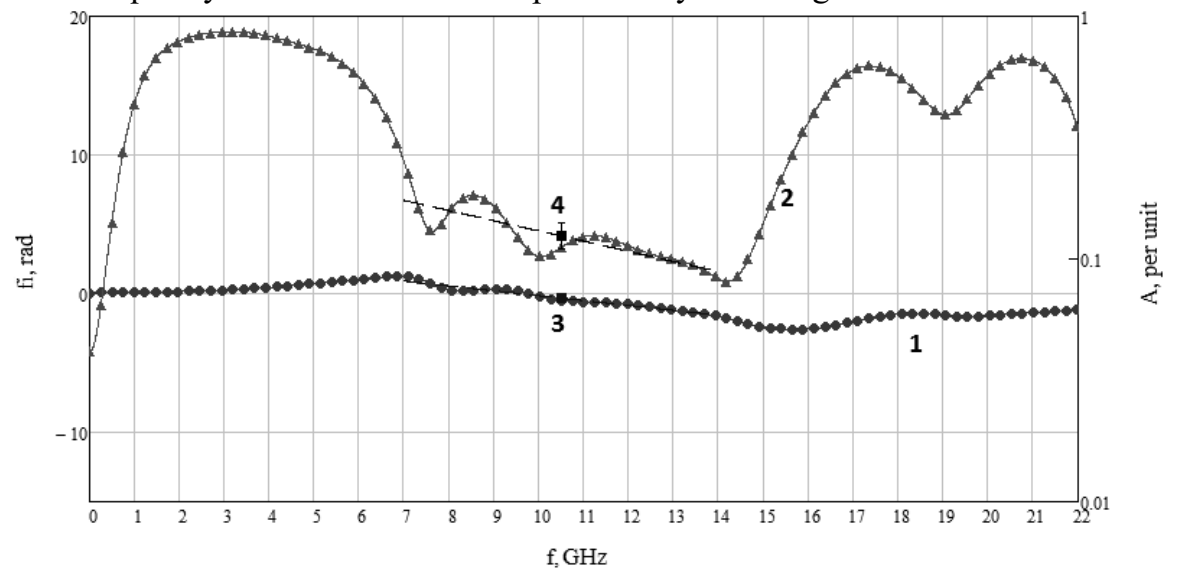

Fig. 4. Dependence of amplitude (2) and phase (1) of transmitted signal on frequency for one layer of rings $3 \mathrm{~cm}$ thick, linear regression of phase (3), linear regression of amplitude (4).

Consider an interval from $7 \mathrm{GHz}$ to $14 \mathrm{GHz}$. In this frequency range, there is a smooth decrease in the amplitude of the signal transmitted through the three-layered lattice from the closed conductors. It can be seen that the phase shift decreases with increasing frequency. The complex refractive index for a lattice of three layers of rings is $n=(0.44 \pm 0.05)+\mathrm{i}(0.15 \pm 0.05)$. It is worth noting that by increasing the number of layers, the 
real part of the refractive index increased in absolute value, and the imaginary part decreased and became close in value for the two layers.

Figure 5 shows graphs showing the dependence of the amplitude and phase of the signal passed through a four-layered grid of closed conductors with a total thickness of $4 \mathrm{~cm}$. From $8 \mathrm{GHz}$ to $15 \mathrm{GHz}$, a strong amplitude sagging associated with a large concentration of ring conductors is seen. At this interval, the refractive index is $(n=0.68 \pm 0.05+\mathrm{i}(0.07 \pm 0.04))$.

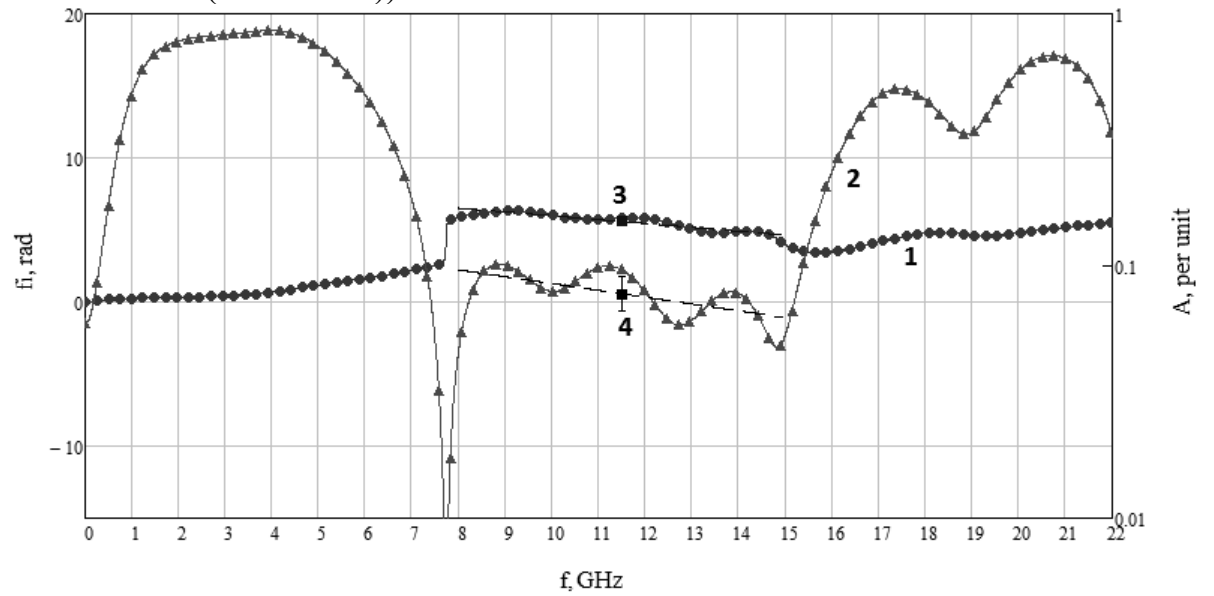

Fig. 5. Dependence of amplitude (2) and phase (1) of transmitted signal on frequency for one layer of rings $4 \mathrm{~cm}$ thick, linear regression of phase (3), linear regression of amplitude (4).

As a result of the experimental study, it can be concluded that such a lattice of annular conductors has a refractive index $n \in[0,1]$.

\section{Radar cross section}

Possible applications of a metamaterial with a negative refractive index have already been discussed. Consider a possible application of a metamaterial with a refractive index $n \in(0$, 1) to reduce radar cross section (RCS) of objects.

The use of a composite material to reduce the RCS targets is illustrated by the example of a coated ball. If the diameter of the ball $R$, and the thickness of the dielectric coating $h$, then for the impact distance $p$, at which the beam of the radio wave, refracted at the boundary, will touch the surface of the metal under the condition (Figure 6) $p=(R+h) \sin \alpha=n R \sin \beta$.

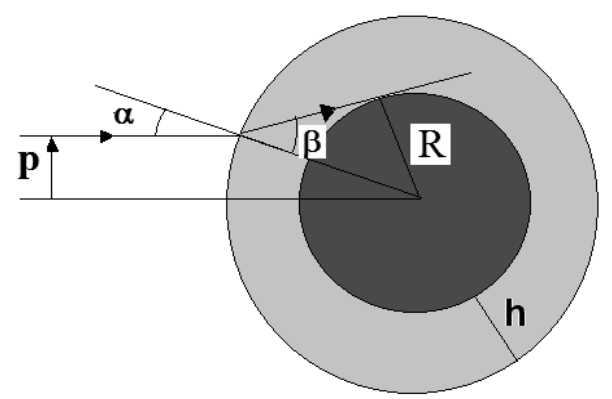

Fig. 6. To the determination of the RSC of a coated ball. 
In this case, the extremely large impact distance, at which the refracted beam will still touch the ball, corresponds to the condition $\sin \beta=R /(\mathrm{R}+\mathrm{h})$. Thus, the greatest sighting distance of the visibility of a coated ball is determined by the condition $p=R n \frac{R}{R+h}$.

Since for an EPR ball is equal to the area of a circle with a radius of an extremely large impact distance, we have for it the relation $S=\pi p^{2}=S_{0}\left(n \frac{R}{R+h}\right)^{2}$, where $S_{0}=\pi R^{2}$ - the cross-section area of the uncoated ball.

The magnitude of the decrease in the RCS of a ball due to a dielectric coating is determined by $G=\frac{S_{0}}{S}=\left(n \frac{R}{R+h}\right)^{2}$. Effective reduction is achieved when $0 \leq n^{2} \leq 1$, that provides the material considered. This will be observed in a sufficiently wide frequency band. When $n^{2}=0$ any object will be invisible. When using conventional dielectric coatings $(1 \leq n)$ or metamaterials $(n \leq-1)$ there will be an increase in RSC.

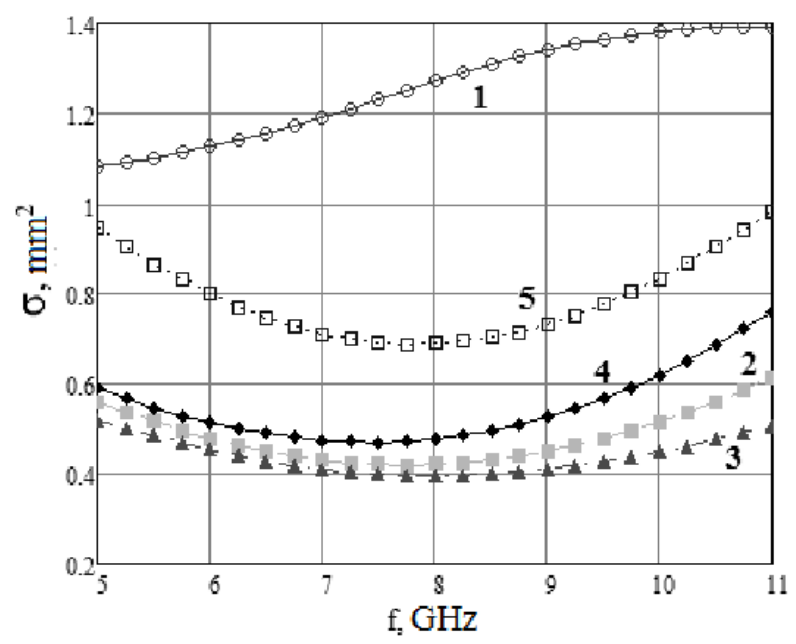

Fig. 7. RSC of the sphere covered with material with an index of refraction:

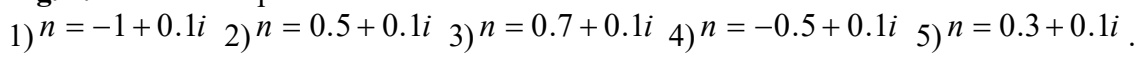

Here are the results of modeling in the software package "CST Microwave studio". As a model, we take a metal ball covered with a material with a different refractive index. The simulation was carried out on the frequency range 5-11 GHz. The RCS values were taken with a frequency step of $0.25 \mathrm{GHz}$. Figure 7 shows the five RCS dependencies ( $\sigma$, MM2) of the frequency, for various refractive indices of the coating material, all the results were normalized to the RCS of a coated ball having a refractive index $n=1$. It can be noted that when $n=-1+0.1 i$ RCS increases in comparison with the metal ball, which is not covered, while the coating of materials $0 \leq n^{2}<1$ reduces the effective scattering area.

\section{Conclusion}

This study shows that the fields scattered by the object covered with a metamaterial with a refractive index $n=0.3+0.1 i$ Similar in structure to fields with scattered spheres with a shell of material $n=-1+0.1 i$, but the RCS of the object is reduced. At the same time, 
the effect of decreasing RCS is more significant when using a coating with a refractive index $0 \leq n<1$, than at $-1<n \leq 0$.

\section{References}

[1] M.I. Finkelstein, Fundamentals of radar. Textbook. for universities. 2 ed. (Radio and Communication, Moscow, 1983)

[2] V.P. Yakubov, A.S. Mironchev, A.G. Andreitsov, I.O. Ponomareva, Artificial Metamaterials and Radio Tomography 53, 859 (2011)

[3] A. Mironchev, A. Gorst, MATEC Web of Conferences 79, 01064 (2016)

[4] A.S. Mironchev, A.V. Klokov, A.V. Gorst, Conference Proceedings of CriMiCo 2014 24th International Crimean Conference Microwave and Telecommunication Technology 1, 702 (2014) 